\title{
Tolerance induction with donor hematopoietic stem cell infusion in kidney transplantation: a single-center experience in China with a 10-year follow-up
}

\author{
Xuanchuan Wang ${ }^{1 \#}$, Cheng Yang ${ }^{1,2 \#} \wedge$, Linkun $\mathrm{Hu}^{1,3}$, Zheng Wei ${ }^{4}$, Qunye Tang ${ }^{1}$, Bing Chen ${ }^{5}$, Yuan $\mathrm{Ji}^{6}$, \\ Ming $\mathrm{Xu}^{1}$, Zhaochong Zeng ${ }^{5}$, Ruiming Rong ${ }^{1,7}$, Tongyu Zhu ${ }^{1}$
}

${ }^{1}$ Department of Urology, Shanghai Key Laboratory of Organ Transplantation, Zhongshan Hospital, Fudan University, Shanghai, China; ${ }^{2}$ Zhangiiang Institute of Fudan University, Shanghai, China; ${ }^{3}$ Department of Urology, The First Affiliated Hospital of Soochow University, Suzhou, China; ${ }^{4}$ Department of Hematology, Zhongshan Hospital, Fudan University, Shanghai, China; ${ }^{5}$ Department of Radiation Oncology, Zhongshan Hospital, Fudan University, Shanghai, China; ${ }^{6}$ Department of Pathology, Zhongshan Hospital, Fudan University, Shanghai, China; ${ }^{7}$ Department of Transfusion, Zhongshan Hospital, Fudan University, Shanghai, China

Contributions: (I) Conception and design: T Zhu, R Rong; (II) Administrative support: Z Wei, Q Tang, B Chen, Y Ji, M Xu, Z Zeng, T Zhu; (III) Provision of study materials or patients: T Zhu; (IV) Collection and assembly of data: X Wang, L Hu; (V) Data analysis and interpretation: X Wang, C Yang; (VI) Manuscript writing: All authors; (VII) Final approval of manuscript: All authors.

\#These authors contributed equally to this work.

Correspondence to: Prof. Tongyu Zhu. Department of Urology, Shanghai Key Laboratory of Organ Transplantation, Zhongshan Hospital, Fudan University, No. 180 Fenglin Road, Shanghai, China. Email: tyzhu_dr@163.com or zhu.tongyu@zs-hospital.sh.cn; Prof. Ruiming Rong. Department of Transfusion, Department of Urology, Shanghai Key Laboratory of Organ Transplantation, Zhongshan Hospital, Fudan University, No. 180 Fenglin Road, Shanghai, China. Email: rong.ruiming@zs-hospital.sh.cn.

Background: Immunosuppressive therapy after life-saving kidney transplantation increases the risk of
infection, cardiovascular diseases, metabolic diseases, and cancer. To date, four centers (three in the USA
and one in South Korea) have reported clinical tolerance trials in kidney transplantation. We performed the
first Chinese clinical trial in which kidney transplantation was combined with donor hematopoietic stem cell
(DHSC) infusion to induce tolerance. This study summarizes the 10-year follow-up results. Methods: From 2009 to 2017, 11 donor/recipient pairs underwent living-related kidney transplantation combined with DHSC infusion. Two of the pairs were human leukocyte antigen (HLA)-matched, and nine were HLA-mismatched. DHSCs were mobilized using granulocyte colony-stimulating factor (G-CSF) and harvested 1 day before transplantation. The recipients received consecutive total lymphoid irradiation (TLI) for 3 days before kidney transplantation. The induction drug was anti-thymocyte globulin (ATG). DHSCs were infused on days 2, 4, and 6 post surgery. All patients were followed-up until Dec 2019. Routine laboratory examinations, chimerism, biopsies, and mixed lymphocyte reactions were performed.

Results: One HLA-matched recipient had 30-50\% chimerism, while the other patients had less than 1\% chimerism. Recipients had donor-specific hyporesponsiveness (DSH) while sustaining normal reactivity to non-donors in mixed lymphocyte reactions. All recipients were followed up for 717-3,918 days. One recipient lost allograft function, and 10 recipients had stable renal function. None of the 11 recipients developed myelosuppression or graft-versus-host disease (GVHD) post transplantation. Our protocol did not increase the risk of infection. Allograft biopsy confirmed that one patient had mild rejection with Banff grade IA, while the other ten recipients did not develop rejection. Five patients were able to reduce the dose of their immunosuppressive therapy.

Conclusions: Our immune tolerance induction protocol, which used DHSC infusion and TLI, achieved low dose immunosuppression with long-term stable kidney allograft survival in Chinese patients.

^ ORCID: Cheng Yang: 0000-0003-4060-2746; Ruiming Rong: 0000-0003-0537-7045. 
Keywords: Kidney transplantation; immune tolerance; hematopoietic stem cell; chimerism

Submitted Mar 14, 2020. Accepted for publication Sep 11, 2020.

doi: $10.21037 /$ atm-20-2502a

View this article at: http://dx.doi.org/10.21037/atm-20-2502a

\section{Introduction}

Although immunosuppressive therapies and antibodies are continually being developed, the long-term survival of renal allografts is still unsatisfactory (1). The main reason for this is rejection, either acute or chronic. Furthermore, many side effects associated with immunosuppression, such as cardiovascular diseases and metabolic disorders, can be lifethreatening to recipients. Therefore, tolerance induction is always the ultimate goal of organ transplantation.

In 2017, the third meeting of the International Clinical Tolerance Workshop was held in Stanford, California, USA. This meeting summarized the updated progress of studies on kidney and liver transplant tolerance induction. Five centers including Stanford, Northwest/Duke, Massachusetts General Hospital, and Samsung Medical Center, reported their progress in kidney clinical tolerance studies. The patients were human leukocyte antigen (HLA)-matched or HLA-mismatched. Some recipients successfully withdrew immunosuppressive therapy after transplantation (2). Briefly, Northwestern established persistent chimerism with $\mathrm{CD}_{34}{ }^{+}$donor hematopoietic stem cells (DHSCs), T cells, and the facilitation of cell infusion. The Northwestern nonmyeloablative conditioning included fludarabine, cyclophosphamide, and total body irradiation (3). They also performed a separate trial of tolerance induction in HLAmatched patients, with $6 / 15$ patients achieving tolerance (4). Stanford used total lymphoid irradiation (TLI) and antithymocyte globulin (ATG) as conditioning regimens, and infused $\mathrm{CD} 34^{+}$DHSCs, and T cells for immune tolerance induction (5). Massachusetts General Hospital carried out two trials in patients with or without hematologic malignancies. In a trial that enrolled HLA-mismatched patients with non-hematologic malignancy, local lymphoid irradiation, cyclophosphamide, and anti-CD2 monoclonal antibody were used as conditioning regimens. Whole bone marrow was infused to induce tolerance (6). All the results from these leading centers are encouraging; however, adverse events cannot be ignored (2). Furthermore, the modulation of immune tolerance induction still faces many hurdles and difficulties. Whether these protocols are suitable for Chinese populations is currently unknown.

From 2009, we performed the first clinical trial of kidney transplantation combined with DHSC infusion for the induction of immune tolerance in Chinese patients. In 2012, we reported a proteomics study of four patients (7). Here, for the first time, we report the long-term follow-up results of a kidney transplantation clinical tolerance study in a group of Chinese patients. We induced transient chimerism by combining kidney transplantation with the infusion of donor $\mathrm{CD} 4^{+}$selected hematopoietic stem cells.

We present the following article in accordance with the STROBE reporting checklist (available at http://dx.doi. org/10.21037/atm-20-2502a).

\section{Methods}

\section{Patients}

Between April 2009 and December 2019, 11 donor/ recipient pairs of subjects from Zhongshan Hospital, Fudan University were enrolled. The average age of the recipients was $33.2 \pm 5.1$ years, ranging from $22-42$ years. The donors ranged in age from $31-62$ years, with an average age of $50.5 \pm 11.1$ years. Ten recipients received primary and one received secondary renal transplantation.

The inclusion criteria for patients were as follows: eligibility to receive renal transplantation; aged $>18$ years; with negative flow crossmatches; and with panel reactive antibodies (PRAs) $\leq 10 \%$. The exclusion criteria were: $\mathrm{ABO}$ incompatibly; active infection; a history of malignant tumor within 5 years; tuberculosis; or pregnancy. Seven pairs of donors and recipients were parent-child relationships, and four pairs were siblings. Two pairs were fully HLA-matched at six HLA loci, and the others were HLA-mismatched at $1-3$ loci. The study was conducted in accordance with the Declaration of Helsinki (as revised in 2013). The study was approved by the ethics committee of Zhongshan Hospital, Fudan University, and informed consent was obtained from all patients $\{$ No. 2009-060[1]\}. The study was registered in the Chinese Clinical Trial Registry (http://www.chictr.org. cn, ChiCTR-TNC-09000399). The general characteristics 
Table 1 General characteristics of donors and recipients

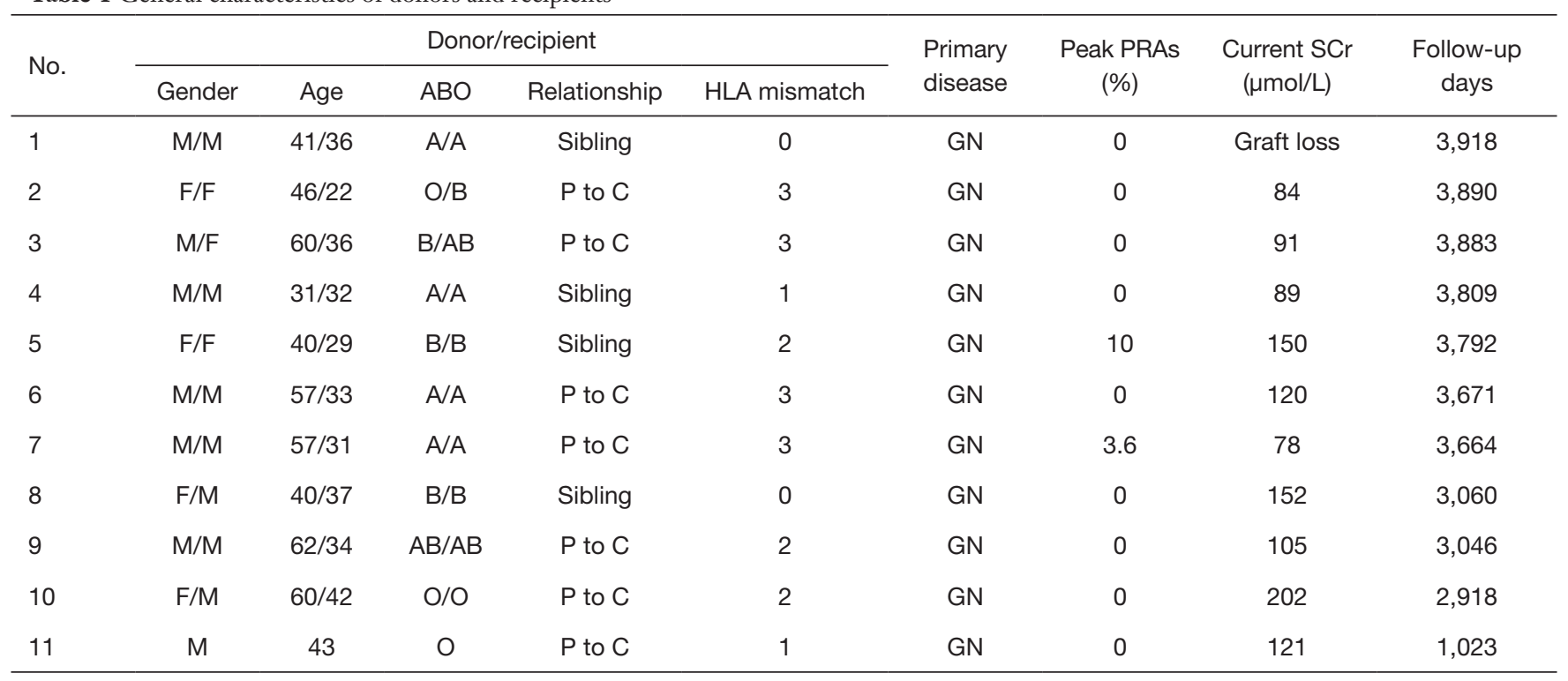

HLA, human leukocyte antigen; PRAs, panel reactive antibodies; $\mathrm{SCr}$, serum creatinine; GN, glomerular nephritis; P to C, parent to child.

Table 2 The total number of infused $\mathrm{CD} 34^{+}$and $\mathrm{CD}^{+}$cells

\begin{tabular}{lcc}
\hline Patient No. & CD34 ${ }^{+}$cells $\left(\times 10^{6} / \mathrm{kg}\right)$ & CD3 $^{+}$cells $\left(\times 10^{8} / \mathrm{kg}\right)$ \\
\hline 1 & 1.69 & 14.39 \\
2 & 0.75 & 0.31 \\
3 & 0.31 & 0.31 \\
4 & 2.35 & 2.98 \\
5 & 0.20 & 0.63 \\
6 & 3.00 & 3.77 \\
7 & 2.17 & 2.0 \\
8 & 0.37 & 2.25 \\
9 & 1.39 & 3.02 \\
10 & 0.22 & 1.73 \\
11 & 0.22 & 1.73 \\
\hline
\end{tabular}

of the donors and recipients are detailed in Table 1.

\section{Clinical protocol}

Before collecting DHSCs, donors received a 5-day course of subcutaneous injections of granulocyte colony-stimulating factor (G-CSF) at a dose of $7.5 \mathrm{mg} / \mathrm{kg} /$ day. G-CSF mobilized donor mononuclear cells into the peripheral blood. These cells were harvested using the COM. TEC blood cell separator (Fresenius AG, Bad Homburg, Germany). $\mathrm{CD} 4^{+}$and $\mathrm{CD}^{+}$cells were assessed using flow cytometry. One day before transplantation, DHSCs were harvested and stored at $-70^{\circ} \mathrm{C}$.

Recipients received TLI on days $-3,-2$, and -1 before kidney transplantation. We used the Siemens ONCOR Impression LINAC System (Siemens, Berlin, Germany), a $6 \mathrm{MV}$ photon beam with a multileaf collimator (MLC), and a pattern of 480-510 cGy/3 Fx/3 D, 160-170 cGy/1 Fx per single fraction. Patients received irradiation to all major lymphatic regions using anterior-posterior-posteroanterior (AP/PA) fields. The supradiaphragmatic or "mantle" field encompassed the low cervical, supraclavicular, infraclavicular, axillary, mediastinal, and pulmonary hilar nodes, as well as the thymus. Adverse events were defined as diarrhea after TLI, graft-versus-host disease (GVHD), severe infection, myelosuppression, and other related symptoms during TLI and DHSC infusion.

During kidney transplantation, recipients received $50 \mathrm{mg}$ of rabbit ATG. On postoperative days (PODs) 2, 4, and 6 , the recipients received an intravenous infusion of cryopreserved DHSCs, which contained CD $34^{+}$cells $(0.2-$ $3.0 \times 10^{6} / \mathrm{kg}$ ) and $\mathrm{CD}^{+}$cells (Table 2). The recipients were treated with a triple immunosuppressive regimen including calcineurin inhibitors (CNIs) (tacrolimus or cyclosporine A), antimetabolic drugs, and corticosteroids (Figure 1). 


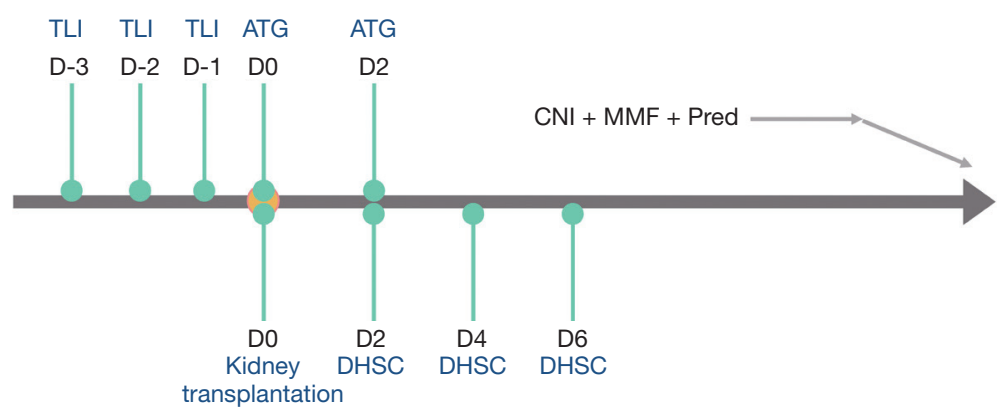

Figure 1 Treatment of recipients. Before kidney transplantation, recipients received TLI 3 times, on days $-3,-2$, and -1 . The induction drug was ATG, which was administered on days 0 and 2. After transplantation, DHSC was infused on days 2, 4, and 6. The maintenance immunosuppression consisted of CNI, MMF and prednisone, which were gradually reduced. TLI, total lymphoid irradiation; ATG, antithymocyte globulin; DHSC, donor hematopoietic stem cell; CNI, calcineurin inhibitor; MMF, mycophenolate mofetil.

\section{Patient follow-up assays}

Chimerism was detected by DNA genotyping of simple sequence-length polymorphic markers that encode short tandem repeats (AmpFl STR Identifiler PCR Amplification Kit, Applied Biosystems, USA). Routine blood examination was performed every day after TLI. The percentage of lymphocyte subsets was measured using flow cytometry. Peripheral blood mononuclear cells (PBMCs) were stained with fluorochrome-labeled anti-CD3, CD4, CD8, CD19, CD56, and CD16 antibodies (eBioscience, San Diego, CA, USA). A mixed lymphocyte reaction was performed to detect $\mathrm{T}$ cell function in recipients. The patients were followed-up once a week in the first 3 months after renal transplantation, every 2 weeks after 3 months, and once a month after half a year. The transplant kidney pathology was assessed by biopsy.

\section{T-cell responses to antigens}

The mixed lymphocyte reaction was performed by culturing peripheral-blood mononuclear cells as responder cells with irradiated donor or unrelated third party allogeneic mononuclear cells as stimulator cells, and measuring $3 \mathrm{H}$-thymidine incorporation. Cells were seeded in roundbottom culture plates and were stimulated with anti-CD3 antibody and soluble anti-CD28 antibody (both from eBioscience) in RPMI 1640 medium, and then incubated for $5-7$ days in a humidified $37^{\circ} \mathrm{C}, 5 \% \mathrm{CO}_{2}$ incubator. Next, $3 \mathrm{H}$-thymidine was added 18 hours before the end of the culture period. The cells were then harvested onto glass fiber mats for the measurement of $3 \mathrm{H}$-thymidine incorporation.

\section{Statistical analysis}

Data are presented as the mean \pm standard deviation. Statistical analysis (SPSS 18.0 software, SPSS, Inc., Armonk, NY, USA) was performed using the two-tailed independent Student's $t$-test (paired or unpaired) after confirming homogeneity of variance using the F-test. Statistical significance was set as $\mathrm{P}<0.05$.

\section{Results}

\section{Donor bone marrow mobilization of hematopoietic stem cells}

Before stem cell harvest, G-CSF $(7.5 \mu \mathrm{g} / \mathrm{kg} / \mathrm{d})$ was administered to donors for 5 consecutive days. The baseline counts of white blood cells (WBCs), neutrophils, lymphocytes, and PBMCs were $6.94 \pm 1.98 \times 10^{9} / \mathrm{L}$, $4.48 \pm 1.92 \times 10^{9} / \mathrm{L}, 1.84 \pm 1.11 \times 10^{9} / \mathrm{L}$, and $0.43 \pm 0.14 \times 10^{9} / \mathrm{L}$, respectively. After mobilization, the average $\mathrm{WBC}$, neutrophil, lymphocyte, and PBMC counts were $41.65 \pm 13.37 \times 10^{9} / \mathrm{L}, 35.79 \pm 11.24 \times 10^{9} / \mathrm{L}, 3.06 \pm 1.12 \times 10^{9} / \mathrm{L}$, and $1.90 \pm 0.69 \times 10^{9} / \mathrm{L}$, respectively. All of the above cell counts significantly increased. The details are shown in Figure 2. In the harvested DHSCs, the total numbers of $\mathrm{CD} 4^{+}$cells and $\mathrm{CD}^{+}$cells were $0.2-3.0 \times 10^{6} / \mathrm{kg}$ and $0.31-$ $14.39 \times 10^{8} / \mathrm{kg}$, respectively (Table 2 ).

\section{Changes in blood cells and lymphocyte subsets after TLI}

After TLI, an immediate and dramatic decrease in lymphocyte count occurred in all patients, reaching 0 after ATG at day 0. Before TLI (day -4), the lymphocyte count was $1.70 \pm 0.61 \times 10^{9} / \mathrm{L}$ with a rapid decrease after 3 days 

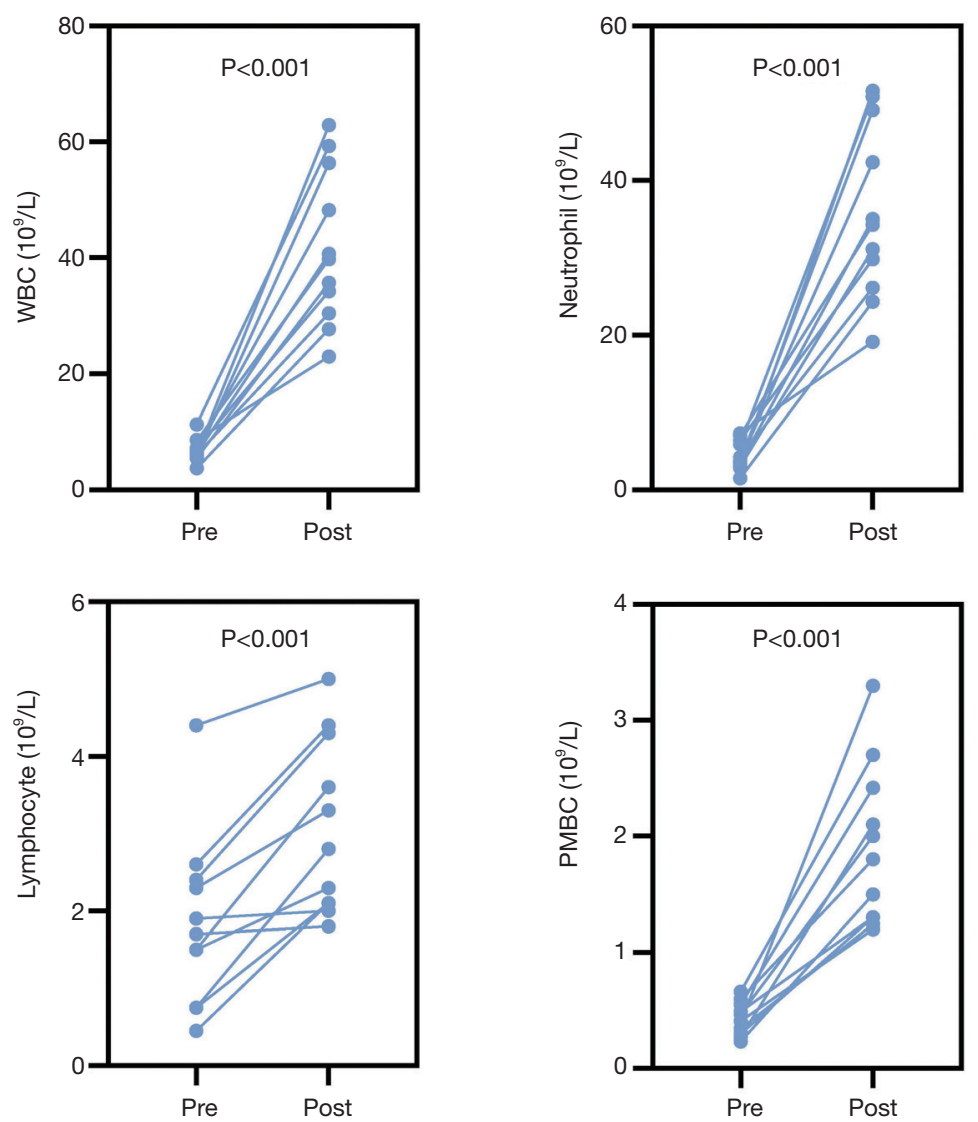

Figure 2 Changes in WBC, neutrophil, lymphocyte, and PBMC cell counts in donors. After bone marrow mobilization, the number of WBCs, neutrophils, lymphocytes and PBMCs significantly increased in donors. WBC, white blood cell; PMBC, peripheral blood mononuclear cell.

(day -1). However, the number of WBCs and neutrophils remained stable. After TLI on day -1 , the proportions

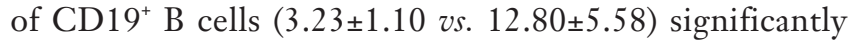
decreased and $\mathrm{CD} 56^{+} \mathrm{CD} 16^{+} \mathrm{NK}$ cells $(10.9 \pm 2.84$ vs. $15.36 \pm 4.70)$ increased compared to counts before TLI (day -1$)$. The percentage of $\mathrm{CD} 8^{+} \mathrm{T}$ cells increased, while $\mathrm{CD}^{+} \mathrm{T}$ cells and $\mathrm{CD} 4^{+} \mathrm{T}$ cells showed no significant change (Table 3). Figure 3 shows each individual data point. All patients had persistent lymphopenia, but their lymphocyte counts gradually recovered after 1 month. The patients' lymphocyte counts increased and returned to normal levels approximately 6 months post transplantation.

\section{Adverse events}

During TLI, one patient experienced fatigue, and five patients had mild diarrhea. During DHSC infusion, one patient exhibited oxygen saturation decline, chest tightness, and discomfort. The symptoms improved after the infusion was stopped. No adverse events were observed in the other recipients. Suspected GVHD was observed in the first patient. After 1 month of follow-up, the skin symptoms disappeared. The other ten recipients did not develop GVHD. The infection risk did not increase compared with that of the routine protocol. No myelosuppression was observed in any of the recipients.

\section{Chimerism induction}

Chimerism was induced in the first recipient who was HLA-matched by 30-50\%, which remained for 4-6 weeks after kidney transplantation (Figure 4). At 6 months, the chimerism had disappeared. The other ten recipients had less than $1 \%$ chimerism (Table 4). 
Table 3 Lymphocyte changes after TLI

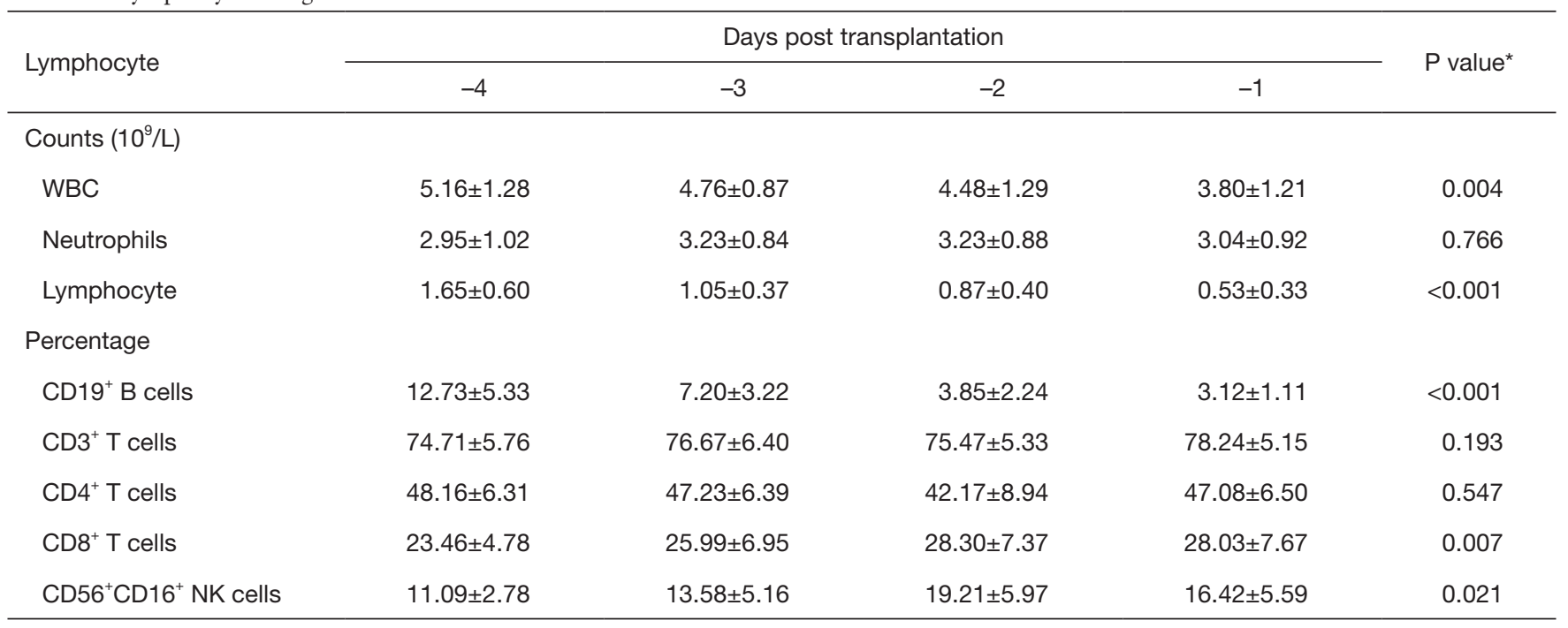

*, comparison between D-4 and D-1. TLI, total lymphoid irradiation; WBC, white blood cell.
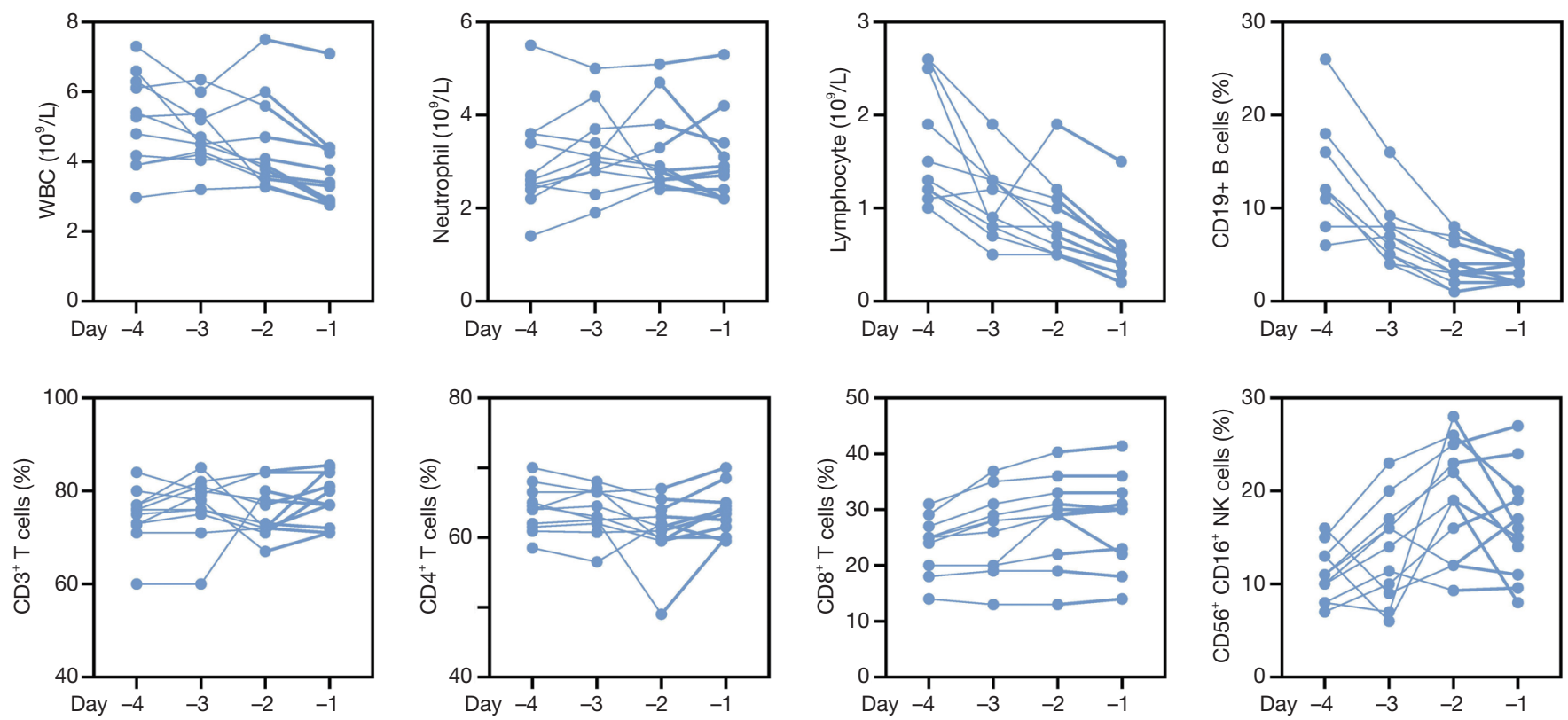

Figure 3 Immune cell changes in recipients after TLI. This figure shows the change in WBC, neutrophil, and lymphocyte counts, as well as T cells, B cells, and NK cells (\% in lymphocytes) before and after TLI. The data at day -4 represents baseline levels before TLI. TLI, total lymphoid irradiation; WBC, white blood cell.

\section{T cell reactivity to donor and non-donor alloantigens}

At 6 months after renal transplantation, we detected the T-cell reactivity of recipients to donor and non-donor alloantigens. Compared to pretreatment, $\mathrm{T}$ cell proliferation in recipients upon donor antigen exposure was significantly reduced post transplantation but remained similar to that after exposure to non-donor antigens. We also set a positive internal control using anti-CD3 and anti-CD28 antibody stimulation. For the external control, we tested T-cell reactivity in a healthy volunteer (Figure 5). These results demonstrated that our protocol induced donor-specific 


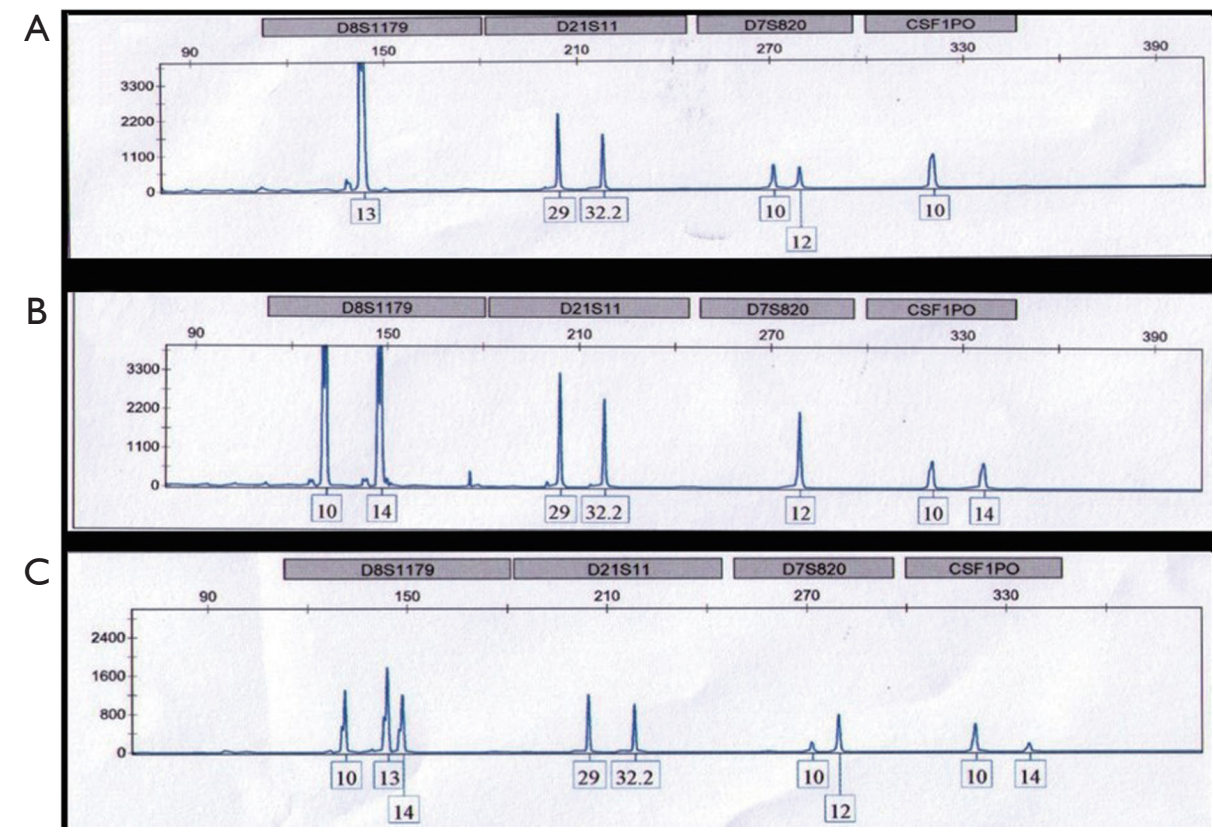

Figure 4 Chimerism on loci D8S1179 in recipient \#1. (A) Loci 13 was observed in the donor; (B) loci 10 and 14 were observed in the recipient before transplantation; (C) 2 weeks after kidney transplantation, loci 13 from the donor was detected in the recipient.

Table 4 Chimerism percentages

\begin{tabular}{lc}
\hline Patient No. & Chimerism \\
\hline 1 & $30-50 \%$, disappeared at 6 months \\
2 & $<1 \%$ \\
3 & $<1 \%$ \\
4 & $<1 \%$ \\
5 & $<1 \%$ \\
6 & $<1 \%$ \\
7 & $<1 \%$ \\
8 & $<1 \%$ \\
9 & $<1 \%$ \\
10 & $<1 \%$ \\
11 & $<1 \%$ \\
\hline
\end{tabular}

hyporesponsiveness (DSH) of T cells in recipients.

\section{Long-term follow-up results}

By December 31, 2019, the median follow-up period was 3,671 days. Nine recipients underwent protocol biopsies $1-3$ years after transplantation. Only one recipient had mild rejection, with Banff grade IA according to the Banff 07 criteria (8), and the remaining ten patients had no rejection (Table 5). The first recipient presented renal allograft dysfunction at 4 years post transplantation. Proteinuria and poor control of hypertension occurred in the fourth year. The biopsy indicated glomerulonephritis relapse without rejection. Therefore, this patient returned to immunosuppressive drug treatment. Unfortunately, he needed to undergo hemodialysis at 4 years after transplantation due to allograft loss. Ten patients had stable allograft function. Among them, five patients reduced the dosage of immunosuppressive agents, four patients used tacrolimus $1.0-3.0 \mathrm{mg} / \mathrm{d}$, and one patient used rapamycin $1 \mathrm{mg} / \mathrm{d}$, mofetil mycophenolate $0.5-1 \mathrm{~g} / \mathrm{d}$, and prednisone $5 \mathrm{mg} / \mathrm{d}$.

\section{Discussion}

In this clinical trial, we induced mixed chimerism in one patient and reduced the dosage of immunosuppressive agents in four patients using DHSC infusion combined with TLI pretreatment. Only one patient experienced mild rejection.

In 2008, the Massachusetts General Hospital of Harvard University and Stanford University were the first institutions to report the successful induction of immune 


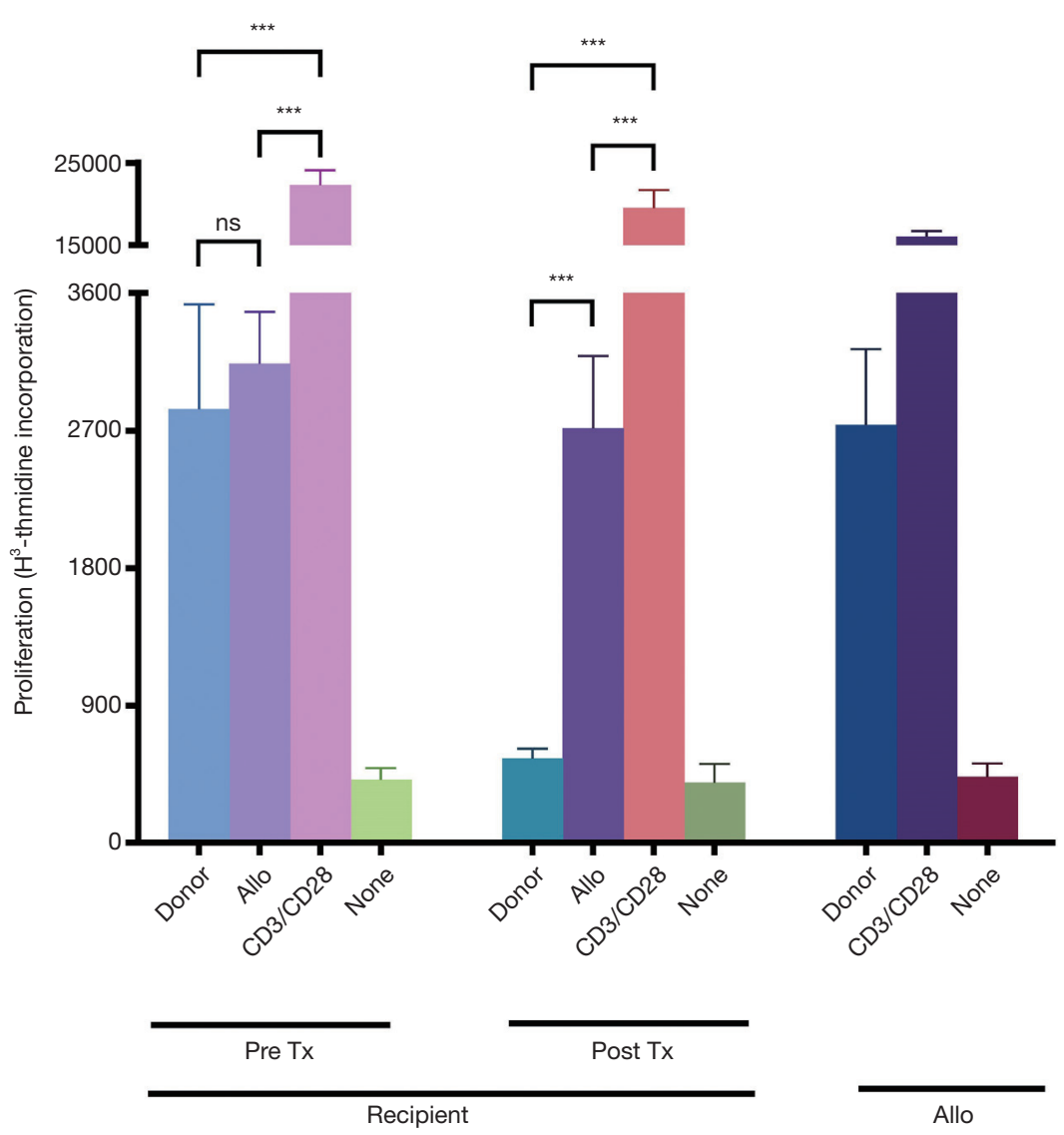

Figure 5 Mixed lymphocyte reactions. This figure represents the T-cell reactivity of patient \#2. Before kidney transplantation and pretreatment, recipient T-cell proliferation significantly increased upon donor or third-party alloantigen stimulation, but after transplantation, T-cell proliferation upon donor antigen stimulation significantly decreased compared to that of the third-party alloantigen, suggesting a low immune response of $\mathrm{T}$ cells specifically to the donor. To test internal quality control, we examined other $\mathrm{T}$ cells from a healthy volunteer. These $\mathrm{T}$ cells demonstrated reactivity after exposure to both the donor and third party alloantigens. CD3/CD28 stimulation was used as a positive control. ${ }^{* * *}, \mathrm{P}<0.001$.

tolerance in living kidney transplantation $(9,10)$. In 2012, Northwestern University reported their clinical trial result of immune tolerance induction using hematopoietic stem cells combined with promoting cells (FCRx) in living kidney transplantation (11). At Massachusetts General Hospital, operational tolerance was induced in seven out of ten patients, four of whom remained off immunosuppressive therapy $(9,12)$. The Stanford University protocol found that 24/29 HLA-identical living donor kidney transplant recipients withdrew immunosuppressive therapy. However, this protocol has not been successful in HLA-mismatched donor/recipient pairs $(5,13)$. At Northwestern University, 26/37 HLA-mismatched, unrelated and related living donor kidney transplant recipients were removed from immunosuppressive therapy $(11,14)$.
Our protocol was similar to that of Stanford University, but the donor hematopoietic cell product did not control for the dose of $\mathrm{CD} 34^{+}$and $\mathrm{CD} 3^{+}$cells. We used TLI 3 days before kidney transplantation. Each dose was 160-170 cGy, and the total dose over 3 days was 480-510 cGy. However, Stanford University used TLI after kidney transplantation (80-120 cGy × 10 times, a total of 800-1,200 cGy) $(5,13)$. As we were the first to carry out a clinical study on kidney transplantation immune tolerance induction in China, we integrated the irradiation protocols of Harvard and Stanford and developed our own protocols for safety and efficacy. Utilizing the phenomena of immune reeducation of autologous or allogeneic hematopoietic stem cell transplantation in autoimmune diseases and leukemia (15-19), we infused a small dose of hematopoietic stem cells 
Table 5 Pathology results of kidney biopsy

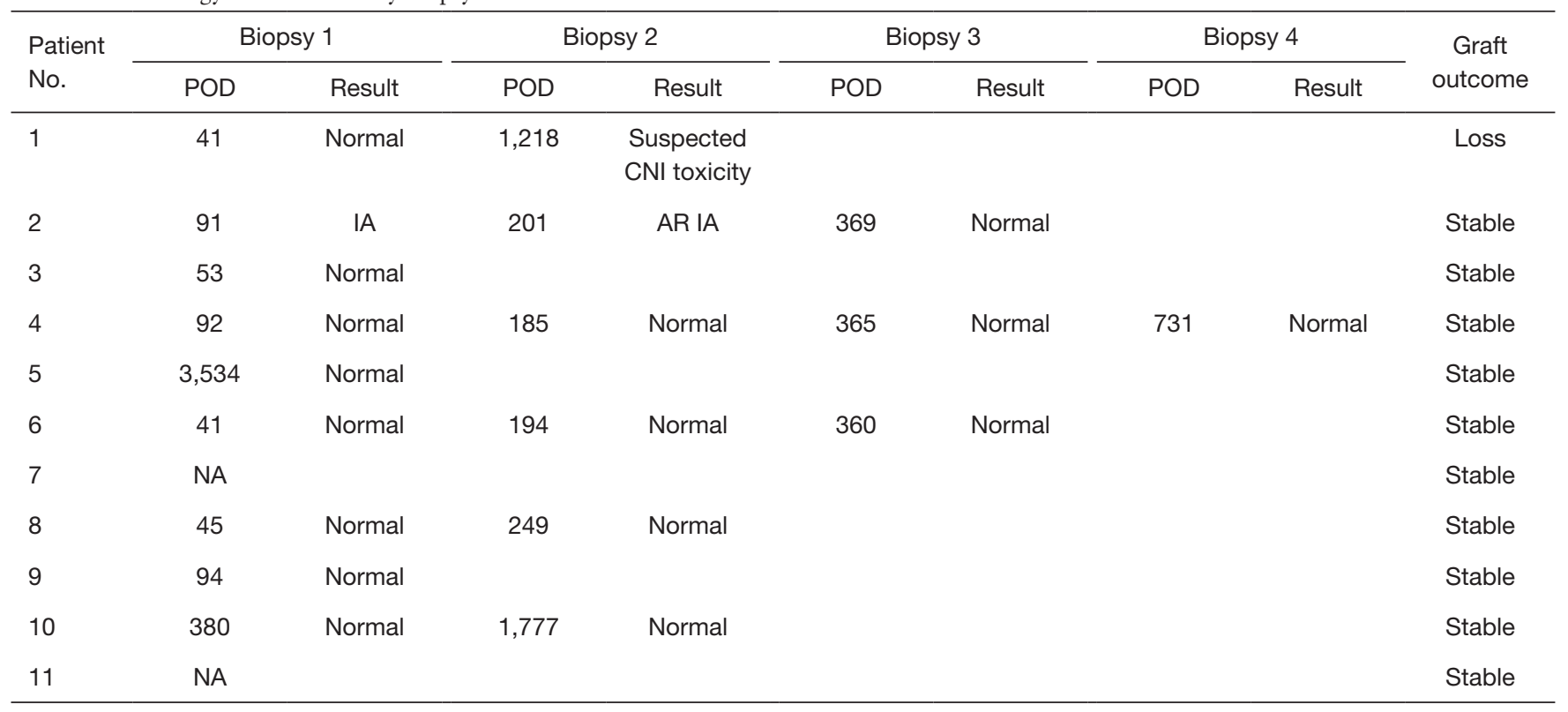

POD, postoperative day; CNI, calcineurin inhibitor; AR, acute rejection.

three times to avoid GVHD. Our long-term follow-up results showed that none of the patients developed GVHD.

Successful induction of chimerism is widely believed to be essential for the induction of immune tolerance (13,20-22), and the three major centers in the United States also induced different degrees of chimerism. Harvard University induced transient chimerism (6), Stanford University induced mixed chimerism, and Northwestern University induced complete chimerism $(3,23,24)$. The Stanford University program successfully achieved continuous mixed chimerism in HLAmatched living kidney transplantation and completely withdrew immunosuppressive drugs. This protocol has also successfully achieved continuous mixed chimerism in HLA-mismatched living kidney transplantation; however, complete discontinuation of immunosuppressive drugs has not yet been achieved. Although immune tolerance was not induced in HLA-mismatched recipients, they found that persistent mixed chimerism prevented both acute and chronic rejection $(5,13)$.

In our study, two donors were HLA-matched, nine were HLA haplotype-matched, and one recipient had $30-50 \%$ induced chimerism. Unfortunately, chimerism disappeared after half a year. Further, the chimeric rate of the other recipients was low. This may be the reason complete immune tolerance was not induced. The dose of $\mathrm{CD} 34^{+}$cells in our protocol was $0.2-3.0 \times 10^{6} / \mathrm{kg}$, which was much lower than that in the Stanford protocol. In their first cohort of six HLA-mismatched patients, the dose of CD34 $4^{+}$ cells was $3.1-11.1 \times 10^{6} / \mathrm{kg}$, and none of the six patients developed chimerism that persisted beyond 3 months. In the second cohort of 22 HLA-matched patients, more CD34 $4^{+}$ cells $\left(4.3-17.5 \times 10^{6} / \mathrm{kg}\right)$ were infused. There were 16 patients who developed chimerism for at least 6 months and had immunosuppressive drugs successfully discontinued without reinstitution, seven had stable chimerism during and after immunosuppressive drug withdrawal, and nine eventually lost chimerism. This HLA-matched cohort had the highest chimerism induction rate. In their third cohort of ten HLA haplotype-matched patients, the dosage of $\mathrm{CD} 34^{+}$cells was the highest at $8-22 \times 10^{6} / \mathrm{kg}$, which was nearly $8-10$ folds higher than our protocol. Half of these patients had induced chimerism (5). However, at Northwestern, the trial enrolled 37 HLA-mismatched patients (one at Duke) and 26 patients achieved durable chimerism ( $\mathrm{T}$ cell chimerism $>50 \%$ ). Twenty-three patients developed "full" peripheral blood chimerism, with $>98 \%$ donor cells (14).

Although our first patient achieved chimerism, most of the patients in our study failed. A relatively low dose of $\mathrm{CD} 34^{+}$cells might be a contributing factor towards this failure. Stable chimerism has been considered a key factor for tolerance induction. Since our chimerism induction failed, we cannot deny the contribution of TLI to the long-term allograft survival with a low dose of immunosuppression. However, we also cannot deny 
the contribution of DHSC infusion. The underlying mechanisms are complex. Although durable donor chimerism is sufficient to establish tolerance, it also indicates that sustained chimerism is by no means absolutely necessary (25). We must aim to achieve a balance between GVHD and chimerism. Northwestern investigated gene expression and microRNA expression profiles in renal biopsy samples from tolerance-induced bioengineered stem cell product (FCRx) recipients, paired donor organs before implantation, and subjects under standard immunosuppression with and without acute rejection (AR) (26). They found some intragraft molecular pathways characteristics, although it was still too early to clarify the full mechanisms. Further trials to find an appropriate dose of DHSCs in Chinese patients are still needed. Besides chimerism, we also found the DSH phenomenon in recipients. Interestingly, DSH alone without successful donor chimerism might not be a predictor of immunosuppression weaning and tolerance. This is consistent with a previous study reported by Leventhal et al. (27).

The ATG dose in our protocol was relatively low compared with the corresponding doses used at the three US centers. These centers also used cyclophosphamide, antiCD2 antibody, and fludarabine. We used unmanipulated hematopoietic stem cells; the number of $\mathrm{CD} 4^{+}$cells infused was lower, but the number of $\mathrm{CD}^{+}$cells was higher. Stanford University controlled hematopoietic stem cell transplantation with CD $34^{+}$cells $\left(10 \times 10^{6} / \mathrm{kg}\right)$ and $\mathrm{CD}^{+}$cells $\left(1 \times 10^{6} / \mathrm{kg}\right)$. Our long-term follow-up results showed that 11 patients did not have GVHD or severe infection, and two patients had slight leukopenia for long-term followup, suggesting that our TLI protocol is safe. Therefore, it is feasible to increase the strength of pretreatment, including the TLI and/or ATG dose.

The risks of our protocol are mainly infection and myelosuppression. To prevent infection, all recipients were placed in laminar airflow after kidney transplantation (for approximately 1 week in the first five patients). Next, we attempted to reduce the time in laminar airflow to 2-3 days. Only one recipient had pulmonary infection. In this study, the first patient had recurrence of nephritis and loss of renal function 4 years after kidney transplantation. At Northwestern University, 2/10 recipients had nephritis relapse in HLA identical living donor transplantation with serial infusion of CD $34^{+}$-selected DHSCs $(23,28)$. However, in another trial using facilitating cells, disease recurrence was not observed in any of the 26 patients where durable chimerism was achieved without immunosuppressive drugs (14). We believe relapse depends on the level of immune system reconstruction and reeducation in recipients; however, the mechanism still needs to be explored. Although the remaining ten recipients were followed up for stable allograft function, we did not perform donor specific antibody (DSA) monitoring. In the future, we should monitor DSA in all recipients.

\section{Conclusions}

This is the first report of immune tolerance induction after kidney transplantation using DHSC infusion in Chinese patients. Although most patients did not achieve chimerism induction, and no patients were able to stop immunosuppressive therapy, all patients reduced their immunosuppression dosage with stable allograft function. Our protocol still needs improvement and adjustment in terms of changing DHSC dosage and frequency in future studies. We will also perform further follow-up of these patients to gain longer-term insight. Using the knowledge gained from this study, we hope to establish a more effective protocol for immune tolerance induction in Chinese patients.

\section{Acknowledgments}

Funding: This study was supported by the National Natural Science Foundation of China (81800659 to XW, 81770746 to CY, 81770747 and 81970646 to RR), National Key R\&D Program of China (2018YFA0107502 to CY, 2018YFA0107501 to RR), Shanghai RisingStar Program (19QA1406300 to CY), the Medical and Health Talents Training Plan for the Excellent Youth of Shanghai Municipal (2018YQ50 to CY), Project ELITE: A Special Supportive Program for Organ Transplantation by COTDF (2019JYJH05 to CY), 2019 Shanghai Youth Talent Development Program (to CY), and the Science and Technology Commission of Shanghai Municipality (16431902300 to TZ).

\section{Footnote}

Reporting Checklist: The authors have completed the STROBE reporting checklist. Available at http://dx.doi. org/10.21037/atm-20-2502a

Data Sharing Statement: Available at http://dx.doi. org/10.21037/atm-20-2502a 
Peer Review File: Available at http://dx.doi.org/10.21037/ atm-20-2502a

Conflicts of Interest: All authors have completed the ICMJE uniform disclosure form (available at http://dx.doi. org/10.21037/atm-20-2502a). Dr. XW reports grants from the National Natural Science Foundation of China during the conduct of the study. Dr. CY reports grants from the National Natural Science Foundation of China, National Key R\&D Program of China, Shanghai Rising-Star Program, Medical and Health Talents Training Plan for the Excellent Youth of Shanghai Municipal, 2019 Shanghai Youth Talent Development Program, and Project ELITE: A Special Supportive Program for Organ Transplantation by COTDF during the conduct of the study. Dr. RR reports grants from the National Natural Science Foundation of China and the National Key R\&D Program of China during the conduct of the study. Dr. TZ reports grants from the Science and Technology Commission of Shanghai Municipality during the conduct of the study. The other authors have no conflicts of interest to declare.

Ethical Statement: The authors are accountable for all aspects of the work in ensuring that questions related to the accuracy or integrity of any part of the work are appropriately investigated and resolved. The study was conducted in accordance with the Declaration of Helsinki (as revised in 2013). The study was approved by the ethics committee of Zhongshan Hospital, Fudan University, and informed consent was obtained from all patients \{No. 2009-060[1]\}. The study was registered in the Chinese Clinical Trial Registry (http://www.chictr.org.cn, ChiCTRTNC-09000399).

Open Access Statement: This is an Open Access article distributed in accordance with the Creative Commons Attribution-NonCommercial-NoDerivs 4.0 International License (CC BY-NC-ND 4.0), which permits the noncommercial replication and distribution of the article with the strict proviso that no changes or edits are made and the original work is properly cited (including links to both the formal publication through the relevant DOI and the license). See: https://creativecommons.org/licenses/by-nc-nd/4.0/.

\section{References}

1. Coemans M, Susal C, Dohler B, et al. Analyses of the short- and long-term graft survival after kidney transplantation in Europe between 1986 and 2015. Kidney Int 2018;94:964-73.

2. Kawai T, Leventhal J, Wood K, et al. Summary of the third international workshop on clinical tolerance. Am J Transplant 2019;19:324-30.

3. Leventhal J, Abecassis M, Miller J, et al. Tolerance induction in HLA disparate living donor kidney transplantation by donor stem cell infusion: durable chimerism predicts outcome. Transplantation 2013;95:169-76.

4. Leventhal JR, Mathew JM, Salomon DR, et al. Nonchimeric HLA-identical renal transplant tolerance: regulatory immunophenotypic/genomic biomarkers. Am J Transplant 2016;16:221-34.

5. Scandling JD, Busque S, Shizuru JA, et al. Chimerism, graft survival, and withdrawal of immunosuppressive drugs in HLA matched and mismatched patients after living donor kidney and hematopoietic cell transplantation. Am J Transplant 2015;15:695-704.

6. Kawai T, Sachs DH, Sprangers B, et al. Long-term results in recipients of combined HLA-mismatched kidney and bone marrow transplantation without maintenance immunosuppression. Am J Transplant 2014;14:1599-611.

7. Wu D, Qi G, Wang X, et al. Hematopoietic stem cell transplantation induces immunologic tolerance in renal transplant patients via modulation of inflammatory and repair processes. J Transl Med 2012;10:182.

8. Solez K, Colvin RB, Racusen LC, et al. Banff 07 classification of renal allograft pathology: updates and future directions. Am J Transplant 2008;8:753-60.

9. Kawai T, Cosimi AB, Spitzer TR, et al. HLAmismatched renal transplantation without maintenance immunosuppression. N Engl J Med 2008;358:353-61.

10. Scandling JD, Busque S, Dejbakhsh-Jones S, et al. Tolerance and chimerism after renal and hematopoieticcell transplantation. N Engl J Med 2008;358:362-8.

11. Leventhal J, Abecassis M, Miller J, et al. Chimerism and tolerance without GVHD or engraftment syndrome in HLA-mismatched combined kidney and hematopoietic stem cell transplantation. Sci Transl Med 2012;4:124ra28.

12. Spitzer TR, Sykes M, Tolkoff-Rubin N, et al. Long-term follow-up of recipients of combined human leukocyte antigen-matched bone marrow and kidney transplantation for multiple myeloma with end-stage renal disease. Transplantation 2011;91:672-6.

13. Scandling JD, Busque S, Lowsky R, et al. Macrochimerism and clinical transplant tolerance. Hum Immunol 2018;79:266-71.

14. Leventhal JR, Ildstad ST. Tolerance induction in HLA 
disparate living donor kidney transplantation by facilitating cell-enriched donor stem cell Infusion: the importance of durable chimerism. Hum Immunol 2018;79:272-6.

15. Down JD, White-Scharf ME. Reprogramming immune responses: enabling cellular therapies and regenerative medicine. Stem Cells 2003;21:21-32.

16. Talmadge JE, Singh R, Ino K, et al. Mechanisms of immune dysfunction in stem cell transplantation. Int $\mathbf{J}$ Immunopharmacol 2000;22:1041-56.

17. Ullah MA, Hill GR, Tey SK. Functional reconstitution of natural killer cells in allogeneic hematopoietic stem cell transplantation. Front Immunol 2016;7:144.

18. Picardi A, Mengarelli A, Marino M, et al. Up-regulation of activating and inhibitory NKG2 receptors in allogeneic and autologous hematopoietic stem cell grafts. J Exp Clin Cancer Res 2015;34:98.

19. Abrahamsson S, Muraro PA. Immune re-education following autologous hematopoietic stem cell transplantation. Autoimmunity 2008;41:577-84.

20. Zuber J, Sykes M. Mechanisms of Mixed Chimerism-Based Transplant Tolerance. Trends Immunol 2017;38:829-43.

21. Sasaki H, Oura T, Spitzer TR, et al. Preclinical and clinical studies for transplant tolerance via the mixed chimerism approach. Hum Immunol 2018;79:258-65.

22. Yolcu ES, Shirwan H, Askenasy N. Mechanisms of tolerance induction by hematopoietic chimerism: the immune

Cite this article as: Wang $\mathrm{X}$, Yang $\mathrm{C}, \mathrm{Hu} \mathrm{L}$, Wei Z, Tang Q, Chen B, Ji Y, Xu M, Zeng Z, Rong R, Zhu T. Tolerance induction with donor hematopoietic stem cell infusion in kidney transplantation: a single-center experience in China with a 10-year follow-up. Ann Transl Med 2020;8(21):1378. doi: $10.21037 / \mathrm{atm}-20-2502 \mathrm{a}$ perspective. Stem Cells Transl Med 2017;6:700-12.

23. Leventhal JR, Mathew JM, Salomon DR, et al. Genomic biomarkers correlate with HLA-identical renal transplant tolerance. J Am Soc Nephrol 2013;24:1376-85.

24. Leventhal JR, Elliott MJ, Yolcu ES, et al. Immune reconstitution/immunocompetence in recipients of kidney plus hematopoietic stem/facilitating cell transplants. Transplantation 2015;99:288-98.

25. Leventhal JR, Mathew JM. Outstanding questions in transplantation: Tolerance. Am J Transplant 2020;20:348-54.

26. Gallon L, Mathew JM, Bontha SV, et al. Intragraft molecular pathways associated with tolerance induction in renal transplantation. J Am Soc Nephrol 2018;29:423-33.

27. Leventhal J, Abecassis M, Miller J, et al. Chimerism and tolerance without GVHD in mismatched recipients of combined hematopoietic stem cell/kidney transplants: donor-specific hyporeactivity is not a reliable biomarker for tolerance. Blood 2013;122:912.

28. Leventhal JR, Miller J, Mathew JM, et al. Updated follow-up of a tolerance protocol in HLA-identical renal transplant pairs given donor hematopoietic stem cells. Hum Immunol 2018;79:277-82.

(English Language Editors: C. Betlazar-Maseh and J. Reynolds) 